\title{
Intraoral Transmission of Streptococcus mutans by a Dental Explorer
}

\author{
WALTER J. LOESCHE, ${ }^{+}$MONA L. SVANBERG, ${ }^{*}$ and HARRY R. PAPE ${ }^{+}$ \\ ${ }^{+}$Department of Oral Biology and Operative Dentistry, University of Michigan School of Dentistry, Ann \\ Arbor, Michigan 48109, and *Department of Odontologiska Klinken, Gotesborgs Universitet, FACK, \\ 40033 Goteborg 33, Sweden
}

A streptomycin-resistant strain of S. mutans was introduced into the mouth as adherent growth on an artificial fissure $(A F)$. A second $A F$, which was initially sterile, was placed in a crown on the opposite side of the dentition. The labeled strain was not found in 8 initially-sterile $A F s$ which were left in vivo for 2 to 6 days and were not examined with a dental explorer. The labeled strain was detected in 7 of 9 initially-sterile AFs which were probed with the dental explorer.

J Dent Res 58(8):1765-1770, August 1979

\section{Introduction.}

In vitro-grown strains of Streptococcus mutans which carry a streptomycin-resistant marker are difficult to establish in the oral cavity of adult volunteers. ${ }^{1,2,3}$ Edman et al. ${ }^{4}$ successfully implanted streptomycinlabeled $S$. mutans in 2 volunteers by means of dental floss. Svanberg and Loesche ${ }^{3,5}$ used an artificial fissure ${ }^{6,7}$ containing adherent growth of $S$. mutans to reliably establish $S$. mutans in the mouths of 3 volunteers. In the floss and artificial fissure studies, the implanted strains remained localized to the side of implantation, as they could not be detected on tooth surfaces on the opposite side of the mouth. This suggested that, under normal circumstances and over a period of several weeks, the probability of $S$. mutans spreading to the opposite side of the dentition via saliva was remote. ${ }^{5}$ Previously, it was found that a sterile dental explorer, after examining a single tooth, removed approximately 3 to $7 \times 10^{6}$ cultivable bacteria. ${ }^{8}$ At that time, the suggestion was made that the dental explorer could serve to inoculate other teeth in the same mouth with $S$. mutans during a routine dental diagnostic exam. The possibility was

Received for publication August 14, 1978 .

Accepted for publication October 10, 1978 .

Supported by Grants No. DE-03011, DE-02731 and DE-03424 from the National Institute of Dental Research. investigated in this study by placing two artificial fissures (AFs) in the same mouth and examining one fissure and then the other with the same explorer.

\section{Materials and methods.}

Subject. - All experiments were performed in the mouth of one adult male volunteer. This individual had no active decay diagnosed within the past two years, had a DMFS score of 75 , and averaged about $7.8 \times 10^{5}$ colony-forming units (CFU) of $S$. mutans per ml of unstimulated saliva. The upper left second premolar (tooth 13) and the upper right first molar (tooth 3) had been endodontically treated, and had been asymptomatic for at least one year prior to the experimental period. Gold crowns were prepared for these teeth so as to receive small gold inlays containing mylar fissures fabricated according to the methods of Loe et al. ${ }^{6}$ The inlays were secured in the recess of the crown with gutta percha. The subject did not change his diet or oral hygiene habits, or receive antibiotics during the time span of the experiment.

Clinical protocol. - The intraoral spread of $S$. mutans was studied in the following way. A sterile AF was secured in tooth 3 , and, in tooth 13 , an AF containing adherent growth of a streptomycin-resistant strain of $S$. mutans was placed. The initially sterile $\mathrm{AF}$ in tooth 3 was removed after various lengths of time, and after using different clinical protocols. On all occasions, the AF inserted with adherent growth of streptomycin-resistant $S$. mutans remained in situ until the initially sterile AF was removed. Three different protocols were used to study the intraoral spread of the labeled $S$. mutans: 1) No dental explorer examination. A sterile $A F$ was secured in tooth 3 . On eight occasions, this initially-sterile AF was removed after 2 to 6 days in vivo without having been examined with a dental explorer. On four of 
these occasions, the sterile $\mathrm{AF}$ was inserted just before the insertion in tooth 13 of the AF with adherent growth. On three occasions, the AF with adherent growth of the streptomycin-resistant strain had been carried for 2 to 22 days before the insertion of the sterile AF. On one occasion, the AF with adherent growth was inserted two days after the insertion of the sterile AF.

2) Dental explorer examination immediately after insertion of the sterile AF. This protocol was performed on four occasions. Two to 6 days after insertion of the $\mathrm{AF}$ with adherent growth in tooth 13 , a sterile $\mathrm{AF}$ was inserted in tooth 3 . The orifice of the AF with the adherent growth was scratched with a sterile dental explorer, and immediately thereafter, the explorer was used to examine the orifice of the initially-sterile AF in tooth 3 .

3) Delayed dental explorer examination. On four occasions, the originally sterile AF in tooth 3 was kept in situ for 2 to 22 days before the dental explorer examination. In this manner, the AF would be filled with unlabeled, indigenous organisms at the time of the explorer examination. ${ }^{9}$ Two to 9 days after this procedure, the initially sterile AF was removed. The AF containing adherent growth of streptomycinresistant $S$. mutans had been carried in tooth 13 for 2 to 26 days at the time of the dental explorer examination.

The AF with adherent growth of streptomycin-resistant $S$. mutans was inserted in tooth 13 six times during the entire experimental period.

Bacteriological Procedures. - A streptomycin-resistant strain of $S$. mutans, strain $S$, which had been shown to reliably establish in the mouth employing the AF model, ${ }^{5}$ was used in these studies. An AF was cultured overnight with strain $S$ in $10 \mathrm{ml}$ of trypticase soy broth (TSB) containing $0.1 \%$ sucrose, and was then serially transferred in new TSB for three consecutive days. This AF contained approximately $10^{7}$ CFUs of the resistant $S$. mutans strain and will be referred to as the AF with adherent growth.

Unstimulated saliva was collected immediately after the insertion of the $\mathrm{AF}$ with adherent growth and, thereafter, once daily in the morning while these AFs were in vivo. One $\mathrm{ml}$ of saliva was dispersed by 5 seconds of sonification (Branson Model W185D), serially diluted in reduced transport fluid (RTF), ${ }^{10}$ and $0.05 \mathrm{ml}$ aliquots from appro- priate dilutions were plated in duplicate on Mitis salivarius bacitracin agar (MSB) ${ }^{11}$ and on MM10 sucrose agar ${ }^{12}$ with and without $0.2 \mathrm{mg} / \mathrm{ml}$ of streptomycin. The contents of the mylar fissures were placed in $10 \mathrm{ml}$ of RTF and processed in a similar manner. All inoculated agar plates were placed in the anaerobic chamber within 30 minutes after collection, and were incubated at $37^{\circ} \mathrm{C}$ for 48 hours in an atmosphere of $85 \% \mathrm{~N}_{2}, 10 \%$ $\mathrm{H}_{2}$ and $5 \% \mathrm{CO}_{2}$.S. mutans was identified by its characteristic colonial morphology on the described media, and by biochemical tests when colonial identification was questionable. ${ }^{13}$ Total CFU counts were obtained from the MM10 sucrose plates, total $S$. mutans counts from the MSB and MM10 sucrose plates, and counts of strain $S$ from the MM10 sucrose-streptomycin plates.

\section{Results.}

No Dental Explorer Examination. - Sterile AFs were inserted 8 times without being probed by a dental explorer. At their removal, after 2 to 6 days in vivo, approximately $1 \times 10^{7} \mathrm{CFU}$ of streptomycinsensitive bacteria could be recovered. No strain $\mathrm{S}$ isolates were detectable, i.e. $<100$ $\mathrm{CFU}$, although, on all occasions, streptomycin-sensitive isolates of $S$. mutans were present and accounted for 0.1 to $0.4 \%$ of the total AF flora. Strain S was detected in 10 salivary samples collected during these experimental periods.

Dental Explorer Examinations. - Streptomycin-resistant isolates of $S$. mutans were found on 3 of 4 occasions in the AF, when a sterile $\mathrm{AF}$ had been inserted in tooth 3 and then immediately probed with a dental explorer which had just examined tooth 13 (Fig. 1). In the only instance where strain $S$ could not be detected in the probed AF, (Fig. 1a), the AF with adherent growth had a low level of strain S, i.e., $0.27 \%$ of the CFU count, at its removal at day 9 . In the sequence shown in Fig. $1 \mathrm{~b}$, the strain $\mathrm{S}$ isolates accounted for $20,25.6$ and $1.2 \%$ of the total CFU count in the probed AFs. When the AF with adherent growth was removed at day 9 , strain $S$ represented $9.7 \%$ of the CFU count. Salivary levels of strain $\mathrm{S}$ varied from $12,000 \mathrm{CFU} / \mathrm{ml}$ immediately after insertion of the $\mathrm{AF}$ with adherent growth, down to $630 \mathrm{CFU} / \mathrm{ml}$ during this experimental period. 


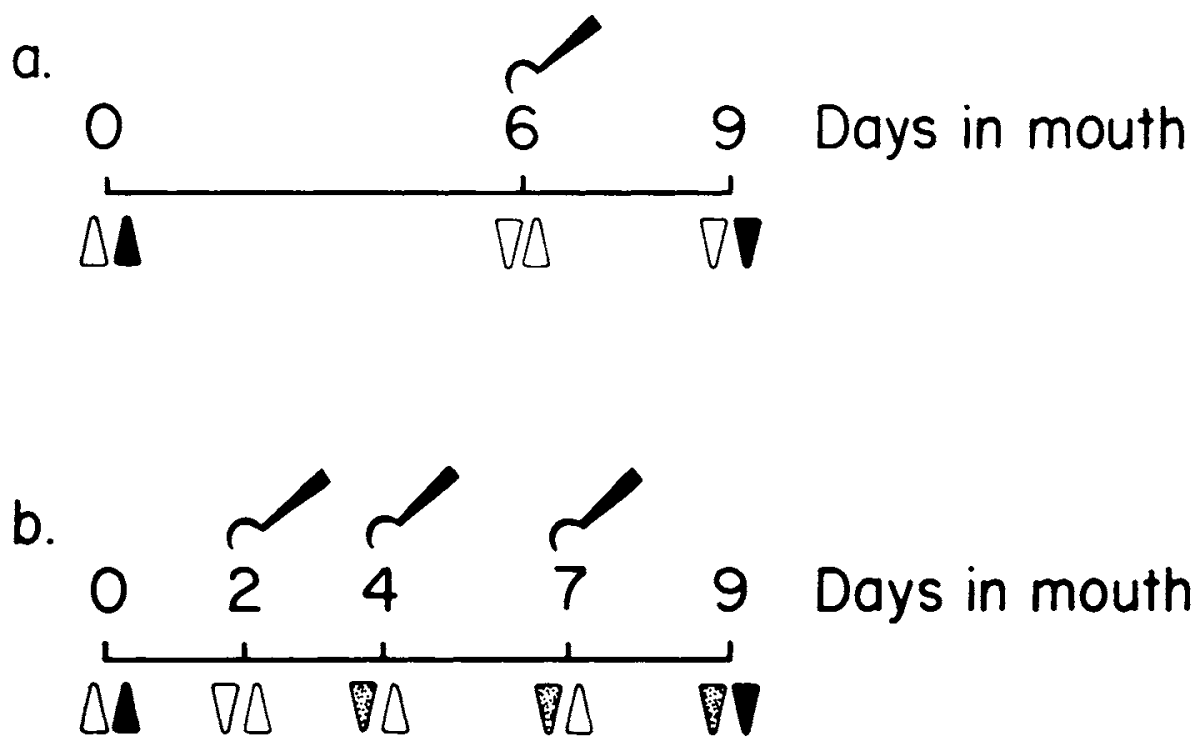

Fig. $1-\Delta S$. mutans adherent artificial fissure placed into tooth $\# 13 ; \Delta$ sterile artificial fissure placed into tooth $\# 3$; $/$ sterile artificial fissure probed with dental explorer; $\nabla$ sterile artificial fissure removed from tooth \#3; $\nabla$ labeled $S$. mutans in sterile artificial fissure removed from tooth $\# 3 ; \nabla S$. mutans adherent fissure removed from tooth \#13.

a.

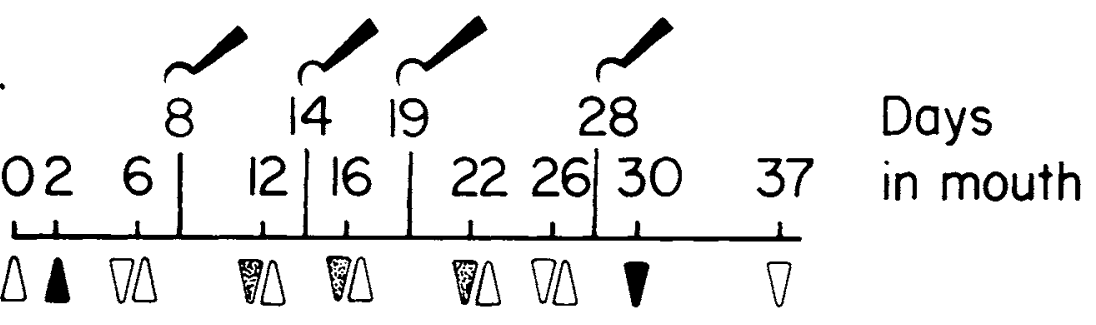

b.

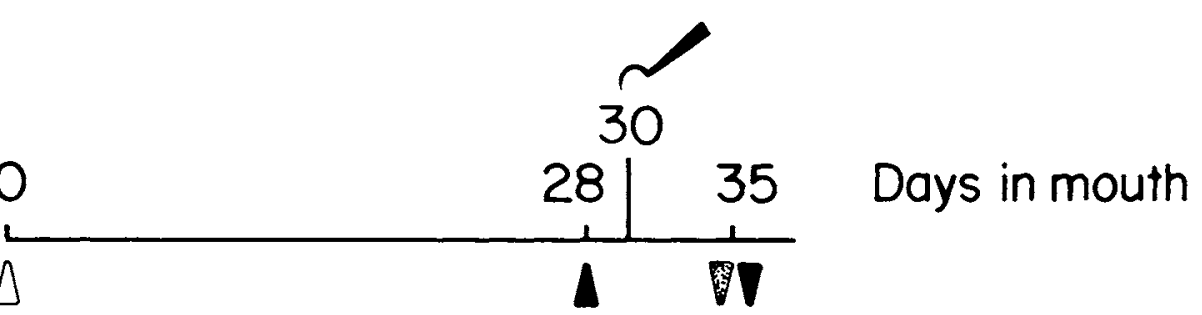

Fig. 2 - $\Delta S$. mutans adherent artificial fissure placed into tooth $\# 13 ; \triangle$ sterile artificial fissure placed into tooth $\# 3 ; \sim$ sterile artificial fissure probed with dental explorer; $\nabla$ sterile artificial fissure removed from tooth $\# 3$; $\nabla$ labeled $S$. mutans in sterile artificial fissure removed from tooth \#3; $S$. mutans adherent fissure removed from tooth \#13. 
TABLE 1

EFFECT OF DENTAL EXPLORER ON THE PRESENCE OF STRAIN S

IN THE INITIALLY STERILE ARTIFICIAL FISSURE (AF)

\begin{tabular}{lcc}
\hline \multicolumn{1}{c}{ Clinical Protocol } & Frequency of Strain S & Proportions \\
\hline No Dental Examination & $0 / 8^{\mathrm{a}}$ & $0.0 \%$ \\
Dental Examination & $3 / 4$ & $15.8 \%$ \\
Delayed Dental Examination & $4 / 5$ & $.006 \%$ \\
\hline
\end{tabular}

aNumerator is number of times strain $\mathrm{S}$ was found in $\mathrm{AF}$; denominator is number of trials.

Delayed Dental Explorer Examination. Sterile AFs placed in tooth 3 remained in vivo 2 to 3 days before they were examined with a dental explorer which had just examined the $\mathrm{AF}$ in tooth 13 . In the sequence shown in Fig. 2a, 3 of 4 probed AFs contained low levels of strain $\mathrm{S}$, i.e., 0.001 to $0.006 \%$ of the total CFU count. Only the sterile $\mathrm{AF}$ inserted at day 26 and probed at day 28 failed to yield detectable levels of strain S. The AF with adherent growth had strain $\mathrm{S}$ accounting for $14.3 \%$ of its CFU at the time of its removal from the mouth, suggesting that an adequate inoculum of strain $S$ was available for explorer transfer. However, the possibility existed that, with passage of time in vivo, strain $\mathrm{S}$ became confined to the lower parts of the AF which were inaccessible to the explorer examination. In a second experiment, a sterile $\mathrm{AF}$ was inserted in tooth 3 and left in situ for 28 days prior to the insertion of an $\mathrm{AF}$ with adherent growth in tooth 13 (Fig. 2b). At day 30 , tooth 13 was examined with an explorer and then tooth 3 was examined. At day 35, both AFs were removed and, in the probed $\mathrm{AF}$, strain $\mathrm{S}$ accounted for $0.002 \%$ of the CFU. Strain $\mathrm{S}$ was undetectable in 5 salivary samples collected during the initial 28 days, but was present in all saliva samples collected while the AF with adherent growth was in situ.

The labeled strain $\mathrm{S}$ was not found in 8 initially-sterile AFs which were left in vivo for 2 to 6 days and which were not examined with a dental explorer (Table 1). However, the labeled strain was detected in 7 of 9 initially-sterile AFs which were probed with the dental explorer (Table 1). If the AFs were sterile at the time of explorer examination, strain $\mathrm{S}$ accounted for about $16 \%$ of the cultivable isolates; if the AFs had been in vivo for 2 or more days, strain $\mathrm{S}$ accounted for only $0.006 \%$ of the isolates (Table 1).

At the time of their removal from the mouth, the initially-sterile AFs were colonized by streptomycin-sensitive strains of $S$. mutans, presumably of indigenous origin, in numbers representing 0.01 to $0.8 \%$ of the total CFU. The salivary levels of these indigenous $S$. mutans strains averaged $50,000 / \mathrm{ml}$, and their range was 6,300 to $500,000 / \mathrm{ml}$ in the 75 salivary samples collected during the entire experimental period. The salivary levels of strain $S$ were high immediately after the insertion of the $\mathrm{AF}$ with adherent growth of strain $\mathrm{S}$, i.e., 2 to $8 \times 10^{6} \mathrm{CFU} / \mathrm{ml}$, but dropped to below $10^{4} \mathrm{CFU} / \mathrm{ml}$ in the following days.

\section{Discussion.}

The results summarized in Table 1 demonstrate that, in this individual, the labeled strain did not traverse from tooth 13 on the left side to tooth 3 on the right side in the absence of an explorer examination. This is in agreement with previous reports 4,5 which noted the unilateral spread of labeled $S$. mutans strains, and can be partially explained by the dilution in saliva of the labeled organisms to numbers which were below the colonization threshold for a tooth surface. ${ }^{5}$ These numbers are in the vicinity of $1,000 \mathrm{CFU} / \mathrm{ml}$ of sonified saliva for fissure surfaces ${ }^{9}, 14$ and $10,000 \mathrm{CFU} / \mathrm{ml}$ for smooth surfaces. ${ }^{15}$ Salivary levels of strain $\mathrm{S}$ were above these colonization thresholds only in the hours following insertion of the $\mathrm{AF}$ with adherent growth. However, these salivary levels were derived from dispersed, whole saliva which would contain contributions from the left side salivary flow which would be enriched for the labeled strain $S$, and from the right side flow which would be low or absent in strain $S$. Thus, the actual levels of strain $\mathrm{S}$ in the local saliva flowing 
over the AF in tooth 3 could be considerably lower than the average values reported above.

This one-sided localization of $S$. mutans suggests that the initial $S$. mutans colonization of human teeth may be unilateral and that the bi-lateral presence of $S$. mutans in a given dentition may reflect: 1) two or more independent colonization events; 2 ) the passage of enough time to raise the probability of $S$. mutans colonization from the saliva; or 3 ) the intervention of an external event(s) in the microecology of the mouth. An examination with a dental explorer could serve as such an external event. The explorer, after examining a single carious tooth, is contaminated with about $500,000 \mathrm{CFU}$ of $S$. mutans. ${ }^{8}$ Some of these CFUs could be deposited on any tooth subsequently examined. In this investigation, the explorer served as a means for transferring strain $S$ from tooth 13 to tooth 3 in 7 of 9 trials (Table 1). In 3 instances, the transfer occurred to an empty AF which had space to accommodate more than $10,000,000 \mathrm{CFU}$ of bacteria. In these cases, strain $S$ accounted for about 16 percent of the CFU recovered from the AF. While these experiments served to show that an explorer examination could spread strain $S$ between the examined teeth, the situation of having an empty AF available for colonization is unrealistic in vivo. Therefore, the experimental design was changed, in that sterile AFs were placed in vivo 2 to 3 days prior to the explorer examination. In this situation, the available space in the AF would be filled with unlabeled, indigenous organisms. When these AFs were subsequently examined by the explorer, only their orifices would be exposed to any bacteria present on the explorer. In this design, strain $S$ colonized the AF in 4 of 5 trials, but its proportions were quite low (Table 1). Whether this small influx of exogenous bacteria could persist and become dominant in the AF flora cannot be ascertained from this experiment. Other investigations indicate that $S$. mutans appears to be selected for the succession which occurs in the AF. ${ }^{9,16}$

These data support the conjecture that the dental explorer could serve as a means for the intraoral transmission of bacteria, in this case $S$. mutans, from tooth to tooth within a given mouth. A similar conclusion was reached by Bielak, ${ }^{17}$ who found a significant increase in the number of tooth sites positive for $S$. mutans following a dental examination. Bergman and Linden 18 postulated from in vitro data that an explorer examination could be caries-provoking, at least on interproximal surfaces, because it might break through the mineralized surface layer and reach the decalcified subsurface enamel. Our studies suggest that, in addition, the explorer could inoculate caries-prone surfaces with an organism which is an overt dental pathogen in animal models ${ }^{19}$ and is significantly associated with human caries. ${ }^{20}$

\section{Conclusion.}

In the single individual studied, the labeled strain did not traverse from tooth 13 on the left side to tooth 3 on the right side in the absence of a dental explorer examination. The labeled strain was detected in 7 of 9 initially sterile AFs which were probed with the dental explorer. The data support the conjecture that the dental explorer could serve as a means for the intraoral transmission of bacteria, in this case $S$, mutans, from tooth to tooth within a given mouth.

\section{REFERENCES}

1. KRASSE, B.; EDWARDSSON, S.; SVENSSON, I.; and TRELL, L.: Implantation of Caries-Inducing Streptococci in the Human Oral Cavity, Archs Oral Biol, 12:231-236, 1967.

2. JORDAN, H. V.; ENGLANDER, H. R.; ENGLER, W. A.; and KULCYZK, S.: Observations on the Implantation and Transmission of Streptococcus Mutans in Humans. $J$ Dent Res, 51:515-518, 1972.

3. SVANBERG, M. and LOESCHE, W. J.: Implantation of Streptococcus Mutans in Man. Archs Oral Biol, 23:551-556, 1978.

4. EDMAN, D. C.; KEENE, H. J.; SHKLAIR, I. L.; and HOERMAN, K. C.: Dental Floss for Implantation and Sampling of Streptococcus Mutans from Approximal Surfaces of Human Teeth. Archs Oral Biol, 20:145$148,1975$.

5. SVANBERG, M. and LOESCHE, W. I.: Intraoral Spread of Streptococcus Mutans in Man. Archs Oral Biol, 23:557-561, 1978.

6. LÖE, H.; KARRING, T.; and THEILADE, E.: An in vivo Method for the Study of Microbiology of Occlusal Fissures. Caries Res, 7:120-129, 1973. 
7. THEILADE, E.; LARSEN, R. H.; and KARRING, T.: Microbiological Studies of Plaque in Artificial Fissures Implanted in Human Teeth. Caries Res, 7:130-138, 1973.

8. LOESCHE, W. J.; WALENGA, A.; and LOOS, P.: Recovery of Streptococcus Mutans and Streptococcus Sanguis from a Dental Explorer after Clinical ixamination of Single Teeth. Archs Oral Biol, 18:571-575, 1973.

9. SVANBERG, M. and LOESCHE, W. J.: The Salivary Concentration of Streptococcus Mutans and Streptococcus Sanguis and Their Colonization of Artificial Tooth Fissures in Man. Archs Oral Biol, 22:441-447, 1977.

10. SYED, S. A. and LOESCHE, W. J.: Survival of Human Dental Plaque Flora in Various Transport Media. Appl Microbiol, 24:638644., 1972.

11. GOLD, O. C.; JORDAN, H. V.; and VAN HOUTE, J.: A Selective Medium for Streptococcus Mutans. Archs Oral Biol, 18:1357$1364,1973$.

12. LOESCHE, W. J. and SYED, S. A.: The Predominant Cultivable Flora of Carious Plaque and Carious Dentine. Caries Res, 7:201-216, 1973.

13. SHKLAIR, I. L. and KEENE, H. J.: A Biochemical Scheme for the Separation of the Five Varieties of Streptococcus Mutans.
Archs Oral Biol, 19:1079-1081, 1974.

14. DUCHIN, S. and VAN HOUTE, J.: Colonization of Teeth in Humans by Streptococcus Mutans as Related to Its Concentration in Saliva and Host Age. Infect and Immun, 20:120-125, 1978 .

15. VAN HOUTE, J. and GREEN, D. B.: Relationship Between the Concentration of Bacteria in Saliva and the Colonization of Teeth in Humans. Infect and Immun, 9: $624-630,1974$.

16. THOTT, E. K.; FOLKE, L. E. A.; and SVEEN, O. B.: A Microbiologic Study of Human Fissure Plaque. Scand $J$ of Dent Res, $82: 428-436,1974$.

17. BIELAK, R.: The Transferability by a Dental Explorer of Streptococcus Mutans During a Dental Examination. Thesis, University of Nebraska, Lincoln, pg 62, 1975.

18. BERGMAN, G. and LINDEN, L. A.: The Action of the Explorer on Incipient Caries. Svensk Tanklak, A. Re., 62:629-634, 1969.

19. KEYES, P. H.: Research in Dental Caries. $J A D A 76: 1357-1373,1968$.

20. LOESCHE, W. J.; ROWAN, J.; STRAFFON, L. H.; and LOOS, P. J.: The Association of Streptococcus Mutans With Human Dental Decay. Infect and Immun, 11:1252-1260, 1975 .

\section{SPECIAL OFFER TO IADR MEMBERS}

To date, subscriptions to the JOURNAL OF ORAL PATHOLOGY have carried a $20 \%$ discount for members of the American Academy of Oral Pathology and the International Association of Oral Pathologists.

This arrangement is now extended to all members of the International Association for Dental Research for personal orders, but not for use by institutions.

The special I.A.D.R. rate for 1979 is D.kr. 308.00 plus postage D.kr. 24.00 (total US $\$ 63$. --subject to exchange rate fluctuations).

Orders should be sent to the publisher:

MUNKSGAARD International Publishers, Ltd.

$35 \mathrm{~N} \phi$ rre S $\phi$ gade

DK-1 370 Copenhagen K

Denmark

Please indicate that you are an I.A.D.R. member. 